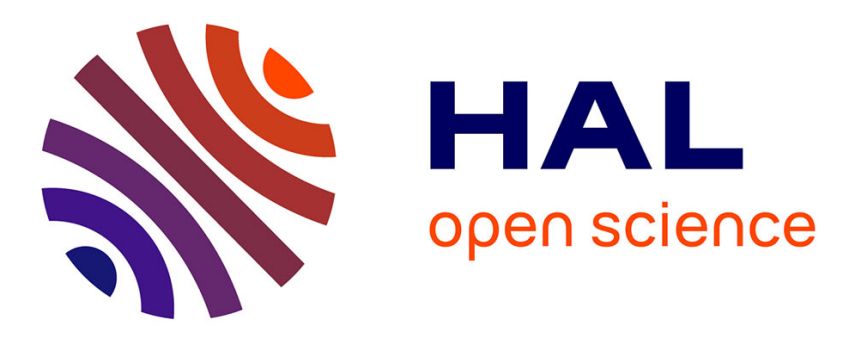

\title{
Analysis of effective elastic properties for shell with complex geometrical shapes
}

\author{
David Guinovart-Sanjuán, Kuppalapalle Vajravelu, Reinaldo \\ Rodríguez-Ramos, Raúl Guinovart-Díaz, Julián Bravo-Castillero, Frédéric \\ Lebon, Federico Sabina
}

\section{To cite this version:}

David Guinovart-Sanjuán, Kuppalapalle Vajravelu, Reinaldo Rodríguez-Ramos, Raúl Guinovart-Díaz, Julián Bravo-Castillero, et al.. Analysis of effective elastic properties for shell with complex geometrical shapes. Composite Structures, 2018, 203, pp.278-285. 10.1016/j.compstruct.2018.07.036 . hal-02021036

\section{HAL Id: hal-02021036 https://hal.science/hal-02021036}

Submitted on 11 Jun 2019

HAL is a multi-disciplinary open access archive for the deposit and dissemination of scientific research documents, whether they are published or not. The documents may come from teaching and research institutions in France or abroad, or from public or private research centers.
L'archive ouverte pluridisciplinaire HAL, est destinée au dépôt et à la diffusion de documents scientifiques de niveau recherche, publiés ou non, émanant des établissements d'enseignement et de recherche français ou étrangers, des laboratoires publics ou privés. 


\title{
Analysis of effective elastic properties for shell with complex geometrical shapes
}

\author{
D. Guinovart-Sanjuán ${ }^{\mathrm{a}, *}$, K. Vajravelu ${ }^{\mathrm{a}}$, R. Rodríguez-Ramos ${ }^{\mathrm{b}}, \mathrm{R}$. Guinovart-Díaz ${ }^{\mathrm{b}}$, \\ J. Bravo-Castillero $^{\mathrm{d}}$, F. Lebon ${ }^{\mathrm{c}}$, F.J. Sabina ${ }^{\mathrm{d}}$ \\ a Department of Mathematics, University of Central Florida, 4393 Andromeda Loop N, Orlando, FL 32816, USA \\ b Departamento de Matemática, Universidad de La Habana, San Lazaro y L, 10400 La Habana, Cuba

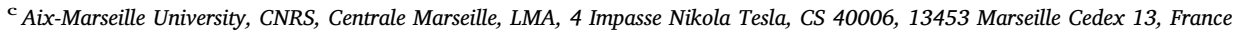 \\ d Instituto de Investigaciones en Matemáticas Aplicadas y en Sistemas, Universidad Nacional Autónoma de México, Delegación Álvaro Obregón, Apartado Postal 20-726, \\ 01000 CDMX, Mexico
}

\begin{abstract}
The manuscript offers a methodology to solve the local problem derived from the homogenization technique, considering composite materials with generalized periodicity and imperfect spring contact at the interface. The general expressions of the local problem for an anisotropic composite with perfect and imperfect contact at the interface are derived. The analytical solutions of the local problems are obtained by solving a system of partial differential equations. In order to validate the model, the effective properties of the structure presented in the literature are obtained as particular cases. The solution of the local problem is used to extend the study to more complex structures, such as, wavy laminates shell composites with imperfect spring type contact at the interface. Also, the results are compared with the results for perfect and imperfect contact models available in the literature.
\end{abstract}

\section{Introduction}

Smarts materials composites present great potential for applications in aerospace, textile and bioengineering industries [1,2]. The development of new technologies in these areas has brought an increase in the use of composite materials and this in turn has brought the expansion and improvement of mathematical and computational methods. One of the main objective of the mathematical and computational methods is the calculation of the effective properties (elasticity, conductivity, etc.) [3-6]. The most common mathematical methods used to compute the effective properties include finite elements method (FEM) [7], Fourier series [8] and multi-scale asymptotic homogenization methods [9-11]. Some authors have used discrete singular convolution method (DSC) for the free vibration analysis of rotating conical shells [12].

Multilayered shells are the most popular composite structures due to their good mechanical properties [13]. Many authors have focused their work on the influence of the geometrical structure of the multilayered composite [14-16]. Also, it have been considered different specific structures, as cylindrical $[17,18]$, spherical $[19,20]$ or truncated conical shell [21]. On the other hand, important studies have been developed in order to see the influence of the contact behavior in the interface of the components on the global properties of the composite [22-24]. The imperfect spring type contact is one of the most widely studied problems. Many authors have been modeling the imperfect contact on fibrous composites with specific geometrical characteristics [25-27].

Many studies have focused their investigation to particular cases of the properties of the composite elements. The most common components are considered isotropic due to its wide appearance in problems of physics and the mechanics of solids [28]. On the other hand, some authors have extended the study of the composite structures to other types of materials (orthotropic, monoclinic, etc). In [10] the asymptotic homogenization method was used to find the effective elastic properties of composite with monoclinic components. According to [29], the DSC reports accurate results for the solution of problems considering orthotropic laminated canonical and cylindrical shells. In [30,31], the authors study the stability of a cylindrical shell composite with components of ceramic, functionally graded materials (FGM) and metal layers. these studies consider the thickness variation for the FGM layer. Some models have presented the thickness variation of the layers as a parametric function of the coordinates [32].

In this contribution, the material coefficients of an elastic

\footnotetext{
* Corresponding author.

E-mail address: guinovart@ucf.edu (D. Guinovart-Sanjuán).
} 
composites are assumed to be rapidly oscillating and periodic functions of a curvilinear coordinates system. The two scales asymptotic homogenization method is used to find the homogeneous problem associated to the equilibrium problem of the system [20,32]. This work gives an approach to analyze the heterogeneous elastic problem in curvilinear structures with general anisotropy, and perfect/imperfect contact at the interface. During the homogenization process, the general expression of the local problems is obtained, considering an generalized periodic anisotropic structure. In previous works, the methods used to solve the local problems were restricted to structures with generalized periodicity but considering perfect contact at the interface [33] or to rectangular laminated composites and isotropic components [11]. As an extension of these contributions, a methodology to solve the local problem for a composite with generalized periodicity, imperfect spring type contact at the interface and anisotropic components is presented. The analytical expression of the local functions are given as a solution of linear equations. In order to validate the present approach, the effective coefficients reported in [10] for a "Chevron" structure with perfect contact at the interface are obtained as special case.The effective coefficients reported in [33] are compared with the results obtained for the imperfect contact case (spring type). As an extension of [34], the effective coefficients of three dimensional wavy laminate composite with imperfect contact at the interface are derived.

The paper is organized as follows. In Section 2, the asymptotic homogenization method is used to derive the general expression of the local problem and the interface conditions. The effective coefficient of a laminate shell composite is introduced in Section 3, where the geometry of the structure is described by a function $\varrho: \mathbb{R}^{3} \rightarrow \mathbb{R}$, [33]. Also, the local problem for anisotropic components of the composite with perfect contact at the interface is obtained as a system of linear equations. In Section 4, the local problem is extended to the case of imperfect contact at the interface (spring type) and the system of partial differential equations associated to the local problem is solved. Finally, the Sections 5, 6 illustrate some examples and applications of the described methodology.

\section{Asymptotic homogenization method for linear curvilinear elastic problem}

In $[32,34]$, the equilibrium elastic problem for a curvilinear composite structure $\Omega=\Omega_{1} \cup \Omega_{2}$, bounded by the surfaces $S_{1}, S_{2}$, is studied. The general expression for the imperfect contact case is given by

$\left.\sigma^{i j}\right|_{j}+f^{i}=0, \quad$ in $\Omega$,

with boundary conditions

$u_{i}=u_{i}^{0} \quad$ on $\quad S_{1}, \quad \sigma^{i j} n_{j}=S_{0}^{i} \quad$ on $\quad S_{2}$,

and interface conditions

$\sigma^{i j} n_{j}=K^{i j}\left[\left[u_{j}\right]\right], \quad$ on $\Gamma$,

$\left[\left[\sigma^{i j} n_{j}\right]\right]=0, \quad$ on $\Gamma$.

Here $\left.(\cdot)\right|_{\mathrm{j}}$ denotes the contravariant derivative, $f^{i}$ is the vector of the body forces, $u_{i}$ is the displacement vector, $n_{j}$ is outward unit normal vector of the surface $S_{2}$ or $\Gamma$ and $u_{i}^{0}$ and $S_{i}^{0}$ are the prescribed values of the displacement and the stress in $S_{1}$ and $S_{2}$, respectively. The surface $\Gamma$ is the interface between the two components of the composite. The matrix $\mathbf{K}=\left[K^{i j}\right]$ characterizes the imperfect contact in $\Gamma$ and the order of $\mathbf{K}$ is $O\left(\varepsilon^{-1}\right)$ and $\llbracket \cdot \rrbracket=(\cdot)^{(2)}-(\cdot)^{(1)}$ denotes the jump at the interface $\Gamma$. In particular case when the components of $\mathbf{K}, K_{i j} \rightarrow \infty$, the problem (1)-(4) reduces to the perfect contact case at the interface.

In order to derive the expression of a homogenized problem associated to (1)-(4), the two-scales asymptotic homogenization method (AHM) is used. In [32,33], a methodology to derive the expression of the following local problems is shown, $\left(\rho_{q, j} C^{i j l k}+\varrho_{p, n} C^{i j m n} N_{m \mid p}^{l k} \varrho_{q, j}\right)_{\mid q}=0, \quad$ on $\quad \mathbf{Y}=\mathbf{Y}_{1} \cup \mathbf{Y}_{2}$,

where $\mathbf{Y}$ is the unit cell, $\mathbf{Y}_{1}, \mathbf{Y}_{2}$ are the components of the unit cell and $\rho=\left(\rho_{1}, \rho_{2}, \rho_{3}\right)$ is the function that described the geometry of the composite.

In [34], the two-scales asymptotic homogenization method is extended to the imperfect contact case and the following general expression of the imperfect spring type interface condition for $N_{m}^{l k}$ was introduced

$\left[\varrho_{q, j} C^{i j l k}+\varrho_{p, n} C^{i j m n} N_{m \mid p}^{l k} \varrho_{q, j}\right](-1)^{\alpha+1} n_{j}=(-1)^{\alpha} K_{i j}\left[\left[N_{j}^{l k}\right]\right]$, on $\Gamma=\overline{\mathbf{Y}}_{1} \cap \overline{\mathbf{Y}}_{2}$,

where $K_{i j} \equiv K_{i j}(\varepsilon)$.

Finally, solving the local problem (5)-(6), the general expression of the homogenized problem is

$\left.\left(C_{e}^{i j k l} v_{k, l}\right)\right|_{j}+f^{i}=0$

$v_{i}=u_{i}^{0} \quad$ on $\quad S_{1}, \quad\left(C_{e}^{i j k l} v_{k} l_{l}\right) n_{j}=S_{i}^{0} \quad$ on $\quad S_{2}$,

where the effective coefficient $\boldsymbol{C}=\left[C_{e}^{i j k l}\right]$ has the following expression by components [32]

$C_{e}^{i j k l}(x)=\left\langle C^{i j k l}+C^{i j m n} \varrho_{p, n} \frac{\partial N_{m}^{k l}}{\partial y_{p}}\right\rangle$.

In the following sections, different techniques are presented in order to solve the local problems (5) and (6) for perfect and imperfect spring contact type case at the interface.

\section{Effective coefficient of a generalized stratified periodic composite with perfect contact condition}

Consider a stratified laminated shell composites, where the periodicity (stratified) function $\rho$ has the property: $\varrho: \mathbb{R}^{m} \rightarrow \mathbb{R}^{1}$ with $m=2,3$ [33].

Now we consider the case when the elastic tensor $\boldsymbol{C} \equiv \boldsymbol{C}\left(\frac{\rho(x)}{\varepsilon}\right)$, and the stratified function $\varrho: \mathbb{R}^{3} \rightarrow \mathbb{R}$, i.e. $\varrho \equiv \rho\left(x_{1}, x_{2}, x_{3}\right)$. Substituting this expression of $\rho$ into (9) and using the Voigt notation, the following equation can be obtained

$$
\begin{aligned}
C_{e}^{a b}= & \left\langle C^{a b}+\left(C^{a 1} \frac{\partial \rho}{\partial x_{1}}+C^{a 6} \frac{\partial \rho}{\partial x_{2}}+C^{a 5} \frac{\partial \rho}{\partial x_{3}}\right) \frac{\partial N_{1}^{b}}{\partial y}\right. \\
& +\left(C^{a 6} \frac{\partial \rho}{\partial x_{1}}+C^{a 2} \frac{\partial \rho}{\partial x_{2}}+C^{a 4} \frac{\partial \rho}{\partial x_{3}}\right) \frac{\partial N_{2}^{b}}{\partial y} \\
& \left.+\left(C^{a 5} \frac{\partial \rho}{\partial x_{1}}+C^{a 4} \frac{\partial \rho}{\partial x_{2}}+C^{a 3} \frac{\partial \rho}{\partial x_{3}}\right) \frac{\partial N_{3}^{b}}{\partial y}\right\rangle .
\end{aligned}
$$

The Eq. (10) is a generalization of the results presented in $[33,11]$ (for instance, see formula (3.35) in [33]).

\subsection{Local problems}

In this section, the local problem for a perfect contact case is solved, i.e. $K^{i j} \rightarrow \infty$ in (6). From (5), the following problems for the local functions $\partial N_{j}^{a} / \partial y$, where $a=1,2,3,4,5,6$ and $j=1,2,3$ are derived in the Voigt's notation [10],

$\frac{\partial}{\partial y}\left[b_{1}^{a}+D_{11} \frac{\partial N_{1}^{a}}{\partial y}+D_{12} \frac{\partial N_{2}^{a}}{\partial y}+D_{13} \frac{\partial N_{3}^{a}}{\partial y}\right]=0$,

$\frac{\partial}{\partial y}\left[b_{2}^{a}+D_{21} \frac{\partial N_{1}^{a}}{\partial y}+D_{22} \frac{\partial N_{2}^{a}}{\partial y}+D_{23} \frac{\partial N_{3}^{a}}{\partial y}\right]=0$,

$\frac{\partial}{\partial y}\left[b_{3}^{a}+D_{31} \frac{\partial N_{1}^{a}}{\partial y}+D_{32} \frac{\partial N_{2}^{a}}{\partial y}+D_{33} \frac{\partial N_{3}^{a}}{\partial y}\right]=0$,

where 
$b_{1}^{a}=\frac{\partial \rho}{\partial x_{1}} C^{1 a}+\frac{\partial \rho}{\partial x_{2}} C^{6 a}+\frac{\partial \rho}{\partial x_{3}} C^{5 a}$,

$b_{2}^{a}=\frac{\partial \rho}{\partial x_{1}} C^{6 a}+\frac{\partial \rho}{\partial x_{2}} C^{2 a}+\frac{\partial \rho}{\partial x_{3}} C^{4 a}$,

$b_{3}^{a}=\frac{\partial \rho}{\partial x_{1}} C^{5 a}+\frac{\partial \rho}{\partial x_{2}} C^{4 a}+\frac{\partial \rho}{\partial x_{3}} C^{3 a}$,

and

$D_{k l}=\frac{\partial \rho}{\partial x_{i}} C^{k i l j} \frac{\partial \rho}{\partial x_{j}}$.

Integrating Eqs. (11)-(13) with respect to the variable $y$ and solving for the local functions $\partial N_{j}^{a} / \partial y$, the following system of equations is obtained

$D_{11} \frac{\partial N_{1}^{a}}{\partial y}+D_{12} \frac{\partial N_{2}^{a}}{\partial y}+D_{13} \frac{\partial N_{3}^{a}}{\partial y}=\lambda_{1}^{a}-b_{1}^{a}$,

$D_{21} \frac{\partial N_{1}^{a}}{\partial y}+D_{22} \frac{\partial N_{2}^{a}}{\partial y}+D_{23} \frac{\partial N_{3}^{a}}{\partial y}=\lambda_{2}^{a}-b_{2}^{a}$,

$D_{31} \frac{\partial N_{1}^{a}}{\partial y}+D_{32} \frac{\partial N_{2}^{a}}{\partial y}+D_{33} \frac{\partial N_{3}^{a}}{\partial y}=\lambda_{3}^{a}-b_{3}^{a}$,

where $\lambda_{j}^{a}$ denotes the integration constant. The system of linear Eqs. (14)-(16) can be written in the matrix form as

$\left[D_{i j}\right]_{3 \times 3} \mathbf{N}_{3 \times 1}^{a}=\lambda_{3 \times 1}^{a}-\mathbf{b}_{3 \times 1}^{a}$,

where $\mathbf{N}_{3 \times 1}^{a}=\left[\frac{\partial N_{1}^{a}}{\partial y}, \frac{\partial N_{2}^{a}}{\partial y}, \frac{\partial N_{3}^{a}}{\partial y}\right]^{T}, \lambda_{3 \times 1}^{a}=\left[\lambda_{1}^{a}, \lambda_{2}^{a}, \lambda_{3}^{a}\right]^{T}$.

The local functions $\partial N_{j}^{a} / \partial y$ are obtained analytically as solutions of the system (17) as follows

$$
\begin{aligned}
\frac{\partial N_{1}^{a}}{\partial y}= & \frac{1}{D}\left[\left(\lambda_{1}^{a}-b_{1}^{a}\right) D_{22} D_{33}+\left(\lambda_{2}^{a}-b_{2}^{a}\right) D_{32} D_{13}+\left(\lambda_{3}^{a}-b_{3}^{a}\right) D_{12} D_{23}\right. \\
& \left.-\left(\lambda_{1}^{a}-b_{1}^{a}\right) D_{23} D_{32}-\left(\lambda_{2}^{a}-b_{2}^{a}\right) D_{12} D_{33}-\left(\lambda_{3}^{a}-b_{3}^{a}\right) D_{13} D_{22}\right],
\end{aligned}
$$

$$
\frac{\partial N_{2}^{a}}{\partial y}=\frac{1}{D}\left[\left(\lambda_{1}^{a}-b_{1}^{a}\right) D_{23} D_{31}+\left(\lambda_{2}^{a}-b_{2}^{a}\right) D_{33} D_{11}+\left(\lambda_{3}^{a}-b_{3}^{a}\right) D_{13} D_{21}\right.
$$$$
\left.-\left(\lambda_{1}^{a}-b_{1}^{a}\right) D_{21} D_{33}-\left(\lambda_{2}^{a}-b_{2}^{a}\right) D_{13} D_{31}-\left(\lambda_{3}^{a}-b_{3}^{a}\right) D_{11} D_{23}\right],
$$

$$
\begin{aligned}
\frac{\partial N_{3}^{a}}{\partial y}= & \frac{1}{D}\left[\left(\lambda_{1}^{a}-b_{1}^{a}\right) D_{21} D_{32}+\left(\lambda_{2}^{a}-b_{2}^{a}\right) D_{31} D_{12}+\left(\lambda_{3}^{a}-b_{3}^{a}\right) D_{11} D_{22}\right. \\
& \left.-\left(\lambda_{1}^{a}-b_{1}^{a}\right) D_{22} D_{31}-\left(\lambda_{2}^{a}-b_{2}^{a}\right) D_{32} D_{11}-\left(\lambda_{3}^{a}-b_{3}^{a}\right) D_{12} D_{21}\right],
\end{aligned}
$$

where $D=\operatorname{det}\left[D_{i j}\right]$.

The local functions depend on the unknown constants $\lambda_{i}^{a}$. In order to find the expression of $\lambda_{i}^{a}$, the average operator is applied in both sides of the Eqs. (18)-(20). Taking into account that $\left\langle\partial N_{j}^{a} / \partial y\right\rangle=0$, the following system of equations for $\lambda_{i}^{a}$ is obtained

$\mathbf{A}_{3 \times 3} \lambda_{3 \times 1}^{a}=\mathbf{B}_{3 \times 1}^{a}$,

where

$\mathbf{A}_{3 \times 3}=\left[\begin{array}{ccc}\left\langle\frac{\left(D_{22} D_{33}-D_{23} D_{32}\right)}{D}\right\rangle & \left\langle\frac{\left(D_{32} D_{13}-D_{12} D_{33}\right)}{D}\right\rangle & \left\langle\frac{\left(D_{12} D_{23}-D_{13} D_{22}\right)}{D}\right\rangle \\ \left\langle\frac{\left(D_{23} D_{31}-D_{21} D_{33}\right)}{D}\right\rangle & \left\langle\frac{\left(D_{33} D_{11}-D_{13} D_{31}\right)}{D}\right\rangle & \left\langle\frac{\left(D_{13} D_{21}-D_{11} D_{23}\right)}{D}\right\rangle \\ \left\langle\frac{\left(D_{21} D_{32}-D_{22} D_{31}\right)}{D}\right\rangle & \left\langle\frac{\left(D_{31} D_{12}-D_{32} D_{11}\right)}{D}\right\rangle & \left\langle\frac{\left(D_{11} D_{22}-D_{12} D_{21}\right)}{D}\right\rangle\end{array}\right]$,

and

$\mathbf{B}_{3 \times 1}$

$$
=\left[\begin{array}{c}
\left\langle b_{1}^{a} \frac{\left(D_{22} D_{33}-D_{23} D_{32}\right)}{D}\right\rangle+\left\langle b_{2}^{a} \frac{\left(D_{32} D_{13}-D_{12} D_{33}\right)}{D}\right\rangle+\left\langle b_{3}^{a} \frac{\left(D_{12} D_{23}-D_{13} D_{22}\right)}{D}\right\rangle \\
\left\langle b_{1}^{a} \frac{\left(D_{23} D_{31}-D_{21} D_{33}\right)}{D}\right\rangle+\left\langle b_{2}^{a} \frac{\left(D_{33} D_{11}-D_{13} D_{31}\right)}{D}\right\rangle+\left\langle b_{3}^{a} \frac{\left(D_{13} D_{21}-D_{11} D_{23}\right)}{D}\right\rangle \\
\left\langle b_{1}^{a} \frac{\left(D_{21} D_{32}-D_{22} D_{31}\right)}{D}\right\rangle+\left\langle b_{2}^{a} \frac{\left(D_{31} D_{12}-D_{32} D_{11}\right)}{D}\right\rangle+\left\langle b_{3}^{a} \frac{\left(D_{11} D_{22}-D_{12} D_{21}\right)}{D}\right\rangle
\end{array}\right] .
$$

Finally, the local functions $\partial N_{j}^{a} / \partial y$ are derived from the Eqs. (18)-(20), once the problem (21) is solved. On the other hand, the

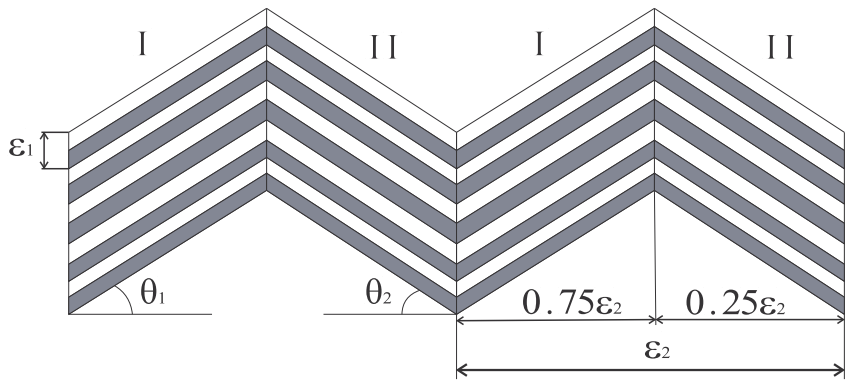

Fig. 1. Chevron structure, two microscale composites with laminate structure, forming angles $\theta_{1}$ and $\theta_{2}$ as is described in [10].

effective coefficients $C_{e}^{a b}$ are computed from (10) using the analytical expressions (18)-(20) for the local functions $\partial N_{j}^{a} / \partial y$.

\subsection{Numerical comparison. "Chevron" structures}

The purpose of this section is to validate the proposed model using the results reported in [10]. In this case, a two-dimensional and twophase laminate with perfect contact at the interface is studied, see Fig. 1. The two main components of this composite are metal, with Young modulus (GPa) 72.4 and Poisson ratio 0.33, and ceramic with 420 Young modulus (GPa) and 0.25 Poisson ratio. The geometry of this structure is described by the function $\rho\left(x_{1}, x_{2}\right)=x_{2}-x_{1} \tan \theta$, where $\theta$ takes the values $\theta_{1}=\pi / 6$ and $\theta_{2}=\pi / 3$ in the regions I and II, respectively.

Now, consider that the fraction volume of the components are $90 \%$ Metal and 10\% Ceramic in regions I and II. Solving the local problem (18)-(20) and substituting the solutions in (10), the following effective coefficients are obtained for region I and II respectively,

$\boldsymbol{C}_{e}^{I}=\left(\begin{array}{cccccc}131.579 & 61.306 & 60.209 & 0 & 0 & 9.403 \\ 61.306 & 117.610 & 57.067 & 0 & 0 & 2.694 \\ 60.209 & 57.067 & 144.373 & 0 & 0 & 2.722 \\ 0 & 0 & 0 & 32.605 & 5.018 & 0 \\ 0 & 0 & 0 & 5.018 & 38.399 & 0 \\ 9.403 & 2.694 & 2.722 & 0 & 0 & 35.518\end{array}\right)$,

$C_{e}^{I I}=\left(\begin{array}{cccccc}117.610 & 61.306 & 57.067 & 0 & 0 & -2.694 \\ 61.306 & 131.579 & 60.209 & 0 & 0 & -9.403 \\ 57.067 & 60.209 & 144.373 & 0 & 0 & -2.722 \\ 0 & 0 & 0 & 38.399 & -5.018 & 0 \\ 0 & 0 & 0 & -5.018 & 32.605 & 0 \\ -2.694 & -9.403 & -2.722 & 0 & 0 & 35.518\end{array}\right)$

After this first step of homogenization, the effective properties of the composite have been derived with different values in region I and II for $\theta_{1}=\pi / 6$ and $\theta_{2}=\pi / 3$, respectively. Now, I and II can be considered as two different materials with their corresponding elastic properties given by (22) and (23), respectively. A new heterogeneous composite is studied using I and II as elements. So, a second homogenization can be used, in order to obtain a global effective coefficient, as described in [10], where I has the $75 \%$ and II has the $25 \%$ of the fraction volume. Here, the effective elastic tensor for the global composite becomes

$\boldsymbol{C}_{e}=\left(\begin{array}{cccccc}127.017 & 60.536 & 59.010 & 0 & 0 & 6.119 \\ 60.536 & 120.330 & 57.505 & 0 & 0 & -0.328 \\ 59.010 & 57.505 & 144.202 & 0 & 0 & 1.303 \\ 0 & 0 & 0 & 33.499 & 2.189 & 0 \\ 0 & 0 & 0 & 2.189 & 36.766 & 0 \\ 6.119 & -0.328 & 1.303 & 0 & 0 & 35.291\end{array}\right)$.

These results coincided with the results reported in [10].

\section{Effective coefficient of a generalized stratified periodic composite with imperfect contact at the interface}

In this section, the solution of the local problem (5) and (6) for a 


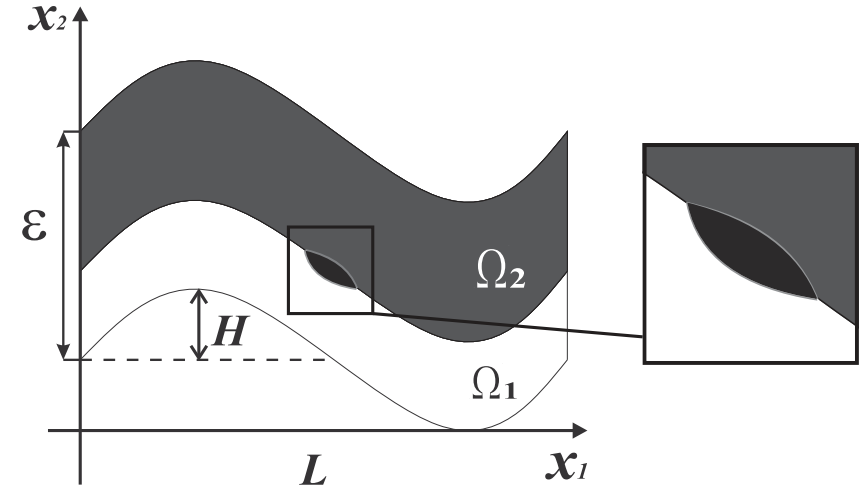

Fig. 2. Unit cell wavy laminate composite with imperfect contact at the interface.

structure with imperfect contact at the interface with stratified generalized periodicity is presented, see Fig. 2. First, consider that $\boldsymbol{C}$ is constant along each component of the unit cell $\mathbf{Y}=\mathbf{Y}_{1} \cup \mathbf{Y}_{2}$. Due to $\partial b_{i}^{a} / \partial y=0$ for all $a=1,2,3,4,5,6$ and $i=1,2,3$, the system (11)-(13) becomes

$D_{11} \frac{\partial^{2} N_{1}^{a}}{\partial y^{2}}+D_{12} \frac{\partial^{2} N_{2}^{a}}{\partial y^{2}}+D_{13} \frac{\partial^{2} N_{3}^{a}}{\partial y^{2}}=0$,

$D_{21} \frac{\partial^{2} N_{1}^{a}}{\partial y^{2}}+D_{22} \frac{\partial^{2} N_{2}^{a}}{\partial y^{2}}+D_{23} \frac{\partial^{2} N_{3}^{a}}{\partial y^{2}}=0$

$D_{31} \frac{\partial^{2} N_{1}^{a}}{\partial y^{2}}+D_{32} \frac{\partial^{2} N_{2}^{a}}{\partial y^{2}}+D_{33} \frac{\partial^{2} N_{3}^{a}}{\partial y^{2}}=0$.

From (25)-(27), we get

$\frac{\partial^{2} N_{i}^{a}}{\partial y^{2}}=0 \Rightarrow N_{i}^{a}= \begin{cases}A_{i}^{a(1)} y+B_{i}^{a(1)} & y \in \mathbf{Y}_{1}, \\ A_{i}^{a(2)} y+B_{i}^{a(2)} & y \in \mathbf{Y}_{2},\end{cases}$

where the upper-scripts $(\cdot)^{(1)},(\cdot)^{(2)}$ represent the value of the function on $\mathbf{Y}_{1}$ and $\mathbf{Y}_{2}$ respectively. Due to the periodicity of the function $N_{i}^{a}$, we have

$N_{i}^{a}\left(y_{1}\right)=N_{i}^{a}\left(y_{2}\right)=0$,

where $y_{1}$ and $y_{2}$ are the boundary surfaces, i.e. $y \in\left[y_{1}, y_{2}\right]$. Therefore $B_{i}^{a(1)}=-A_{i}^{a(1)} y_{1}, \quad B_{i}^{a(2)}=-A_{i}^{a(2)} y_{2}$.

Now, rewriting the imperfect interface condition (6), taking into account the expression of $N_{i}^{a}$ given in (28) and considering that $\varrho: \mathbb{R}^{3} \rightarrow \mathbb{R}$, the following $6 \times 6$ system can be derived

$\left[W_{i j}\right] \mathbf{A}=\mathbf{P}^{a}$,

where $\mathbf{A}=\left[A_{1}^{a(1)}, A_{2}^{a(1)}, A_{3}^{a(1)}, A_{1}^{a(2)}, A_{2}^{a(2)}, A_{3}^{a(2)}\right]^{T}, \mathbf{P}^{a}$ is the vector of independents terms of each equation and $\left[W_{i j}\right]$ are the coefficients of $\mathbf{A}$ in each of the equations, the expression of $\mathbf{P}^{a}$ and $\left[W_{i j}\right]$ are shown in Appendix A. Solving the system (29), the local functions are obtained and substituted into (10), the effective coefficients for imperfect contact at the interface are derived.

\subsection{Numerical comparison. Rectangular laminate composite with imperfect contact}

In order to validate the result above mentioned, a rectangular laminated shell composite is considered as described in section 6.1 of [34], where $\partial \rho / \partial x_{1}=\partial \rho / \partial x_{2}=0$ and $\partial \rho / \partial x_{3}=1$. The unit cell is composed of two layers of isotropic materials, first, aluminum with Young modulus 72.04 and Poisson ratio $=0.3$, the other element is reinforced carbon fiber with Young modulus 150 and Poisson ratio $=0.35$. The volume fraction of each element in the unit cell is $50 \%$. The matrix $\mathbf{K}$ that characterizes the imperfect contact takes the following non- vanishing values $K_{11}=K_{22}=\mu / \varepsilon$ and $K_{33}=(\lambda+2 \mu) / \varepsilon$, where $\lambda=\mu=1$ and $\varepsilon=0.01$, [34]. Substituting these values in (29), the local function are obtained. From (10), the effective coefficient for spring type imperfect contact has a perfect match with the result reported in [34] for rectangular laminates composite.

\section{Effective properties for two-dimensional wavy composite with imperfect contact at the interface}

In [33], a methodology to derive the effective coefficients for a twophase two-dimensional wavy composite is introduced, for a structure with perfect contact at the interface and isotropic components. This procedure was extended in [32] to composite with imperfect contact, where the imperfection is modeled considering a third thin layer between the elements (soft and hard interface). On the other hand, in [34] the two-scales asymptotic homogenization method is used to find the expression of the local problem and the effective coefficient of a laminate composite (no wavy) with spring type contact at the interface and isotropic elements. The generalization of all these works presented in Section 4 for a laminate composite with generalized periodicity, spring type imperfect contact at the interface and anisotropic components is used to derive some particular cases. The effective coefficients obtained using (10), and the solution of the system (29) for a composite with mechanical imperfect contact spring type with generalized coordinates is compared with the perfect contact case reported in [33,32].

Consider two-phase structure where the geometry is described by the function

$\rho\left(x_{1}, x_{2}\right)=x_{2}-H \sin \left(\frac{2 \pi x_{1}}{L}\right)$,

where $H$ is a parameter related to the oscillation, $L$ is the length of the unit cell, [33], (see Fig. 2). The two elements $\Omega_{1}, \Omega_{2}$ of the composite are aluminum with Young modulus $E_{1}=72.04 \mathrm{GPa}$, Poisson ratio $v_{1}=0.35$ (volume fraction 80\%) and stainless steel with Young modulus $E_{1}=206.74 \mathrm{GPa}$, Poisson ratio $\nu_{1}=0.3$ (volume fraction $20 \%$ ), respectively.

An imperfect contact spring type between the layers is considered (see Fig. 2). The matrix $\mathbf{K}$ characterized the imperfection and it has the following expression

$\mathbf{K}=\varepsilon^{-1}\left[\begin{array}{ccc}\mu & 0 & 0 \\ 0 & \mu & 0 \\ 0 & 0 & \lambda+2 \mu\end{array}\right]$

where $\mu=0.8 \mu_{1}+0.2 \mu_{2}, \lambda=0.8 \lambda_{1}+0.2 \lambda_{2} ; \mu_{1}, \lambda_{1}$ and $\mu_{2}, \lambda_{2}$ are the Lame's constant of the elements $\Omega_{1}$ and $\Omega_{2}$ respectively. In order to compare for different values of the matrix $\mathbf{K}$, the parameter $\varepsilon$ takes the following values $[0.05,0.01,0.001]$.

In Fig. 3, a comparison between the effective coefficients $C_{e}^{23}$ and $C_{e}^{66}$ reported in [32] for a laminate wavy composite with perfect contact at the interface and the corresponding coefficients considering mechanical imperfect contact spring type at the interface are shown, for the values of $\varepsilon=[0.05,0.01,0.001]$. The approximation to the perfect contact case is illustrated when $\varepsilon \rightarrow 0$, i.e. $K^{i j} \rightarrow \infty$.

\section{Three-dimensional bi-periodical wavy structures}

As a final application of the present study described in this paper, a general three-dimensional wavy structure is considered, where $\varrho: \mathbb{R}^{3} \rightarrow \mathbb{R}$ and the expression is given by

$\rho\left(x_{1}, x_{2}, x_{3}\right)=x_{3}-H_{1} \sin \left(\frac{2 \pi}{L_{1}} x_{1}\right)-H_{2} \sin \left(\frac{2 \pi}{L_{2}} x_{2}\right)$,

where $H_{1}, H_{2}$ denote the heights of the oscillations in the structure and $L_{1}, L_{2}$ are are the periods lengths of the wavy for $x_{1}$ and $x_{2}$ respectively, (Fig. 4a). The wavy function (32) is a generalization of the structure 


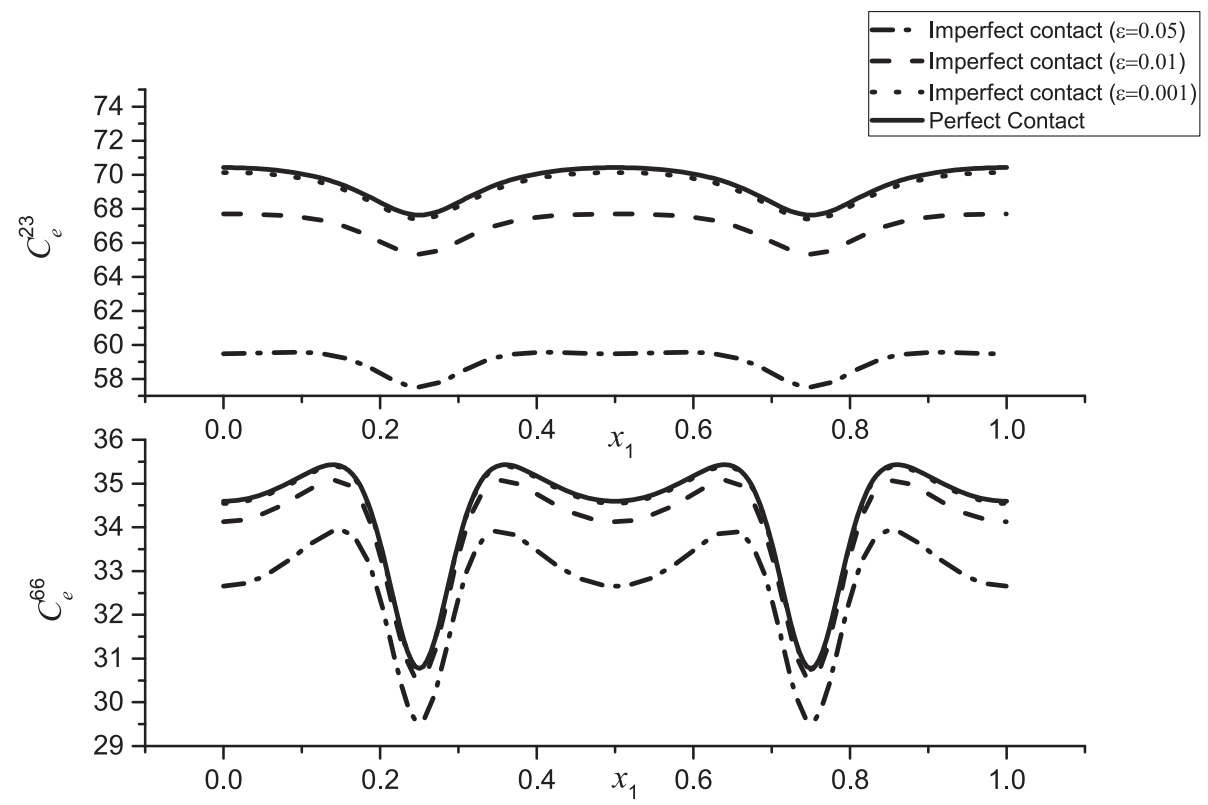

Fig. 3. Comparison of the effective coefficients $C_{e}^{23}$ and $C_{e}^{66}$ for composite with spring type imperfect contact at the interface for $\varepsilon=[0.05,0.01,0.001]$ and the perfect contact case.

composite studied in [33], where the considered geometry for the wavy structure is constant with respect to $x_{2}$ and the oscillation is only regarding to $x_{1}$ (Fig. $4 \mathrm{~b}$ ). The Fig. $4 \mathrm{~b}$ can be derived from (32) taking $\mathrm{H}_{2}=0$.

In order to study the influence of the geometry in the elastic properties, the effective coefficients $C_{e}^{11}$ and $C_{e}^{56}$ are computed, considering spring type imperfect contact at the interface of the composite. The obtained results are compared with the perfect contact case described in Section 3. Two numerical experiments are considered where the isotropic material constituents used are stainless steel (with Young's modulus, $E_{1}=206.74 \mathrm{GPa}$, Poisson ratio, $\nu_{1}=0.3$ ) with thickness $V_{1}=0.2$ (volume fraction) and aluminum (with Young's modulus $E_{2}=72.04 \mathrm{GPa}$, Poisson ratio $\nu_{2}=0.35$ ) with thickness $V_{2}=0.8$. The geometry of the composites are described by (32) with parameters, heights $H_{1}=H_{2}=0.25$ and lengths of the periodicities $L_{1}=L_{2}=1$ for structure Fig. 4a. The example when heights $H_{1}=0.25, H_{2}=0$ and length of the periodicity $L_{1}=1$, Fig. $4 \mathrm{~b}$, is studied in [32].

The matrix that characterize the imperfect contact of the structure is given in (31), with $\varepsilon=1 / 100, K^{11}=K^{22}=\mu$ and $K^{33}=\lambda+2 \mu$, where $\mu=37.25$ and $\lambda=73.66$. In Table 1 and Table 2 the effective coefficients $C_{e}^{11}$ and $C_{e}^{56}$ are computed considering spring type imperfect contact at the interface using the methodology described in Section 4. The obtained results are compared with the perfect contact case at the interface for the points of $\left(x_{1}, x_{2}\right) \in[0,0.15,0.3,0.45]^{2}$.

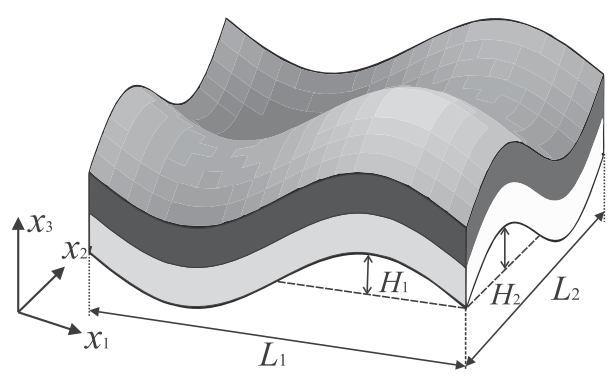

(a) Wavy structure along $x_{1}$ and $x_{2}$.

\section{Conclusions}

In the paper, a methodology to solve the local problem of a laminate shell composite with generalized periodicity, imperfect spring type contact at the interface and anisotropic elements is given. The analytical expression for the local functions are obtained. As an extension of $[33,32]$, the local problem is solved considering a generalized wavy structure, where the components are anisotropic materials. The asymptotic homogenization method is used to derive the results presented in [10] for the case of a "Chevron" structure with perfect contact at the interface. On the other hand, the general expression of the local problem for a structure composed by anisotropic elements, imperfect spring type contact at the interface and with generalized periodicity is derived. For the particular case of laminate shell composite, the local problem was a reduce to a system of $6 \times 6$ of linear equations. The analytical expressions for the local functions are expressed as a solution of the system. Finally, in order to validate the model, the effective coefficient of a three dimensional wavy structure is computed and the results are compared with the results in $[33,32,34]$ as particular cases.

This method allows us to obtain the model equation for the homogenized problem of a wider range of shell structures with generalized periodicity. It allows us to study the elastic equilibrium equation for several structures with imperfect contact between the components. This methodology can also be extended considering variable imperfection along the structure or to another kind of composites as piezoelectric,

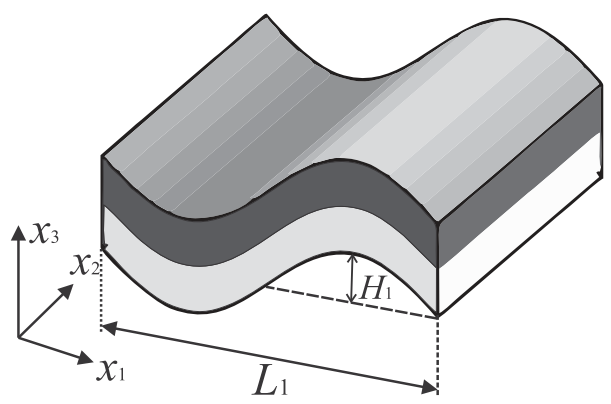

(b) Wavy structure along $x_{1}$.

Fig. 4. Heterogeneous curvilinear wavy laminated structures. 
Table 1

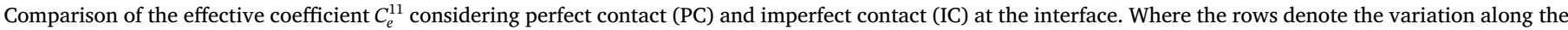
$x_{1}$ direction and the columns in the $x_{2}$ direction.

\begin{tabular}{|c|c|c|c|c|c|c|c|c|}
\hline \multirow{2}{*}{$\begin{array}{l}x_{2} \backslash x_{1} \\
\text { Method }\end{array}$} & \multicolumn{2}{|c|}{0} & \multicolumn{2}{|c|}{0.15} & \multicolumn{2}{|c|}{0.30} & \multicolumn{2}{|c|}{0.45} \\
\hline & PC & IC(100) & PC & IC(100) & PC & IC(100) & PC & IC(100) \\
\hline 0 & 135.23 & 129.66 & 140.10 & 136.85 & 143.97 & 141.82 & 135.68 & 130.41 \\
\hline 0.15 & 132.84 & 127.14 & 137.25 & 134.24 & 142.47 & 140.89 & 133.16 & 127.79 \\
\hline 0.30 & 131.82 & 126.01 & 135.37 & 132.41 & 141.11 & 139.82 & 132.04 & 126.56 \\
\hline 0.45 & 134.92 & 129.34 & 139.81 & 136.59 & 143.83 & 141.76 & 135.36 & 130.09 \\
\hline
\end{tabular}

Table 2

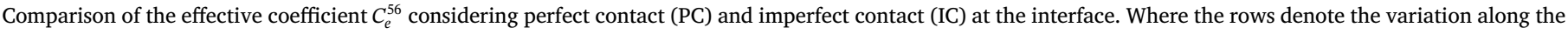
$x_{1}$ direction and the columns in the $x_{2}$ direction.

\begin{tabular}{|c|c|c|c|c|c|c|c|c|}
\hline \multirow{2}{*}{$\begin{array}{l}x_{2} \backslash x_{1} \\
\text { Method }\end{array}$} & \multicolumn{2}{|c|}{0} & \multicolumn{2}{|c|}{0.15} & \multicolumn{2}{|c|}{0.30} & \multicolumn{2}{|c|}{0.45} \\
\hline & PC & IC(100) & $\mathrm{PC}$ & IC(100) & PC & IC(100) & PC & IC(100) \\
\hline 0 & -0.337 & -0.174 & 1.018 & 1.209 & 2.245 & 2.450 & -0.227 & -0.061 \\
\hline 0.15 & -0.891 & -0.777 & 0.206 & 0.350 & 1.934 & 2.096 & -0.838 & -0.721 \\
\hline 0.30 & 0.779 & 0.713 & 0.263 & 0.176 & -1.152 & -1.255 & 0.773 & 0.705 \\
\hline 0.45 & 0.418 & 0.259 & -0.946 & -1.133 & -2.245 & -2.447 & 0.311 & 0.149 \\
\hline
\end{tabular}

thermoelectric, with several applications in civil and mechanic engineering. Furthermore, the study could be extended to nonlinear constitutive laws of the materials and study membranes, which has important applications in biomedicine to study cornea, aorta, skin membranes, etc.

\section{Acknowledgment}

The Authors thank the Editor and the anonymous Reviewers for their valuable comments and suggestions, that contributed to improve the work. The funding of Proyecto Nacional de Ciencias Básicas 2013-2015 (Project No. 7515) are gratefully acknowledged. Thanks to Departamento de Matemáticas y Mecánica IIMAS-UNAM and FENOMEC for their support and Ramiro Chávez Tovar and Ana Pérez Arteaga for computational assistance. R. Rodríguez-Ramos wants to thank the stay at University of Central Florida, Orlando, where the manuscript was prepared and discussed. The authors, Rodríguez-Ramos and Guinovart-Díaz, would like to thank the projects CONACYT - CB2016-01 285241 and PHC Carlos J. Finlay 2018PROJET N39142TA.

\section{Appendix A. Components of the matrix and the vector of the system (29)}

The expressions $\frac{\partial \rho^{*}}{\partial x_{i}}$, for $i=1,2,3$ denote the value of the functions $\frac{\partial \rho}{\partial x_{i}}$ at the interface surface $y^{*}=\Gamma$ and $y_{1}, y_{2}$ denote the boundary surfaces of $\mathbf{Y}_{1}, \mathbf{Y}_{2}$ respectively.

$$
\begin{aligned}
& W_{11}=\left(C^{11(1)} \frac{\partial \rho^{*}}{\partial x_{1}}+C^{16(1)} \frac{\partial \rho^{*}}{\partial x_{2}}+C^{15(1)} \frac{\partial \rho^{*}}{\partial x_{3}}\right) n_{1}+\left(C^{16(1)} \frac{\partial \rho^{*}}{\partial x_{1}}+C^{66(1)} \frac{\partial \rho^{*}}{\partial x_{2}}+C^{56(1)} \frac{\partial \rho^{*}}{\partial x_{3}}\right) n_{2}+\left(C^{15(1)} \frac{\partial \rho^{*}}{\partial x_{1}}+C^{56(1)} \frac{\partial \rho^{*}}{\partial x_{2}}+C^{55(1)} \frac{\partial \rho^{*}}{\partial x_{3}}\right) n_{3}+K_{11}\left(y^{*}-y_{1}\right) \\
& W_{12}=\left(C^{16(1)} \frac{\partial \rho^{*}}{\partial x_{1}}+C^{12(1)} \frac{\partial \rho^{*}}{\partial x_{2}}+C^{14(1)} \frac{\partial \rho^{*}}{\partial x_{3}}\right) n_{1}+\left(C^{66(1)} \frac{\partial \rho^{*}}{\partial x_{1}}+C^{26(1)} \frac{\partial \rho^{*}}{\partial x_{2}}+C^{46(1)} \frac{\partial \rho^{*}}{\partial x_{3}}\right) n_{2}+\left(C^{56(1)} \frac{\partial \rho^{*}}{\partial x_{1}}+C^{25(1)} \frac{\partial \rho^{*}}{\partial x_{2}}+C^{45(1)} \frac{\partial \rho^{*}}{\partial x_{3}}\right) n_{3}+K_{12}\left(y^{*}-y_{1}\right) \\
& W_{13}=\left(C^{15(1)} \frac{\partial \rho^{*}}{\partial x_{1}}+C^{14(1)} \frac{\partial \rho^{*}}{\partial x_{2}}+C^{13(1)} \frac{\partial \rho^{*}}{\partial x_{3}}\right) n_{1}+\left(C^{56(1)} \frac{\partial \rho^{*}}{\partial x_{1}}+C^{46(1)} \frac{\partial \rho^{*}}{\partial x_{2}}+C^{36(1)} \frac{\partial \rho^{*}}{\partial x_{3}}\right) n_{2}+\left(C^{55(1)} \frac{\partial \rho^{*}}{\partial x_{1}}+C^{45(1)} \frac{\partial \rho^{*}}{\partial x_{2}}+C^{35(1)} \frac{\partial \rho^{*}}{\partial x_{3}}\right) n_{3}+K_{13}\left(y^{*}-y_{1}\right) \\
& W_{14}=K_{11}\left(y_{2}-y^{*}\right) \quad W_{15}=K_{12}\left(y_{2}-y^{*}\right) \quad W_{16}=K_{13}\left(y_{2}-y^{*}\right) \\
& W_{21}=K_{11}\left(y^{*}-y_{1}\right) \quad W_{22}=K_{12}\left(y^{*}-y_{1}\right) \quad W_{23}=K_{13}\left(y^{*}-y_{1}\right) \\
& W_{24}=\left(C^{11(2)} \frac{\partial \rho^{*}}{\partial x_{1}}+C^{16(2)} \frac{\partial \rho^{*}}{\partial x_{2}}+C^{15(2)} \frac{\partial \rho^{*}}{\partial x_{3}}\right) n_{1}+\left(C^{16(2)} \frac{\partial \rho^{*}}{\partial x_{1}}+C^{66(2)} \frac{\partial \rho^{*}}{\partial x_{2}}+C^{56(2)} \frac{\partial \rho^{*}}{\partial x_{3}}\right) n_{2}+\left(C^{15(2)} \frac{\partial \rho^{*}}{\partial x_{1}}+C^{56(2)} \frac{\partial \varrho^{*}}{\partial x_{2}}+C^{55(2)} \frac{\partial \rho^{*}}{\partial x_{3}}\right) n_{3}+K_{11}\left(y_{2}-y^{*}\right) \\
& W_{25}=\left(C^{16(2)} \frac{\partial \rho^{*}}{\partial x_{1}}+C^{12(2)} \frac{\partial \rho^{*}}{\partial x_{2}}+C^{14(2)} \frac{\partial \rho^{*}}{\partial x_{3}}\right) n_{1}+\left(C^{66(2)} \frac{\partial \rho^{*}}{\partial x_{1}}+C^{26(2)} \frac{\partial \varrho^{*}}{\partial x_{2}}+C^{46(2)} \frac{\partial \rho^{*}}{\partial x_{3}}\right) n_{2}+\left(C^{56(2)} \frac{\partial \rho^{*}}{\partial x_{1}}+C^{25(2)} \frac{\partial \rho^{*}}{\partial x_{2}}+C^{45(2)} \frac{\partial \rho^{*}}{\partial x_{3}}\right) n_{3}+K_{12}\left(y_{2}-y^{*}\right) \\
& W_{26}=\left(C^{15(2)} \frac{\partial \rho^{*}}{\partial x_{1}}+C^{14(2)} \frac{\partial \rho^{*}}{\partial x_{2}}+C^{13(2)} \frac{\partial \rho^{*}}{\partial x_{3}}\right) n_{1}+\left(C^{56(2)} \frac{\partial \rho^{*}}{\partial x_{1}}+C^{46(2)} \frac{\partial \rho^{*}}{\partial x_{2}}+C^{36(2)} \frac{\partial \rho^{*}}{\partial x_{3}}\right) n_{2}+\left(C^{55(2)} \frac{\partial \rho^{*}}{\partial x_{1}}+C^{45(2)} \frac{\partial \rho^{*}}{\partial x_{2}}+C^{35(2)} \frac{\partial \rho^{*}}{\partial x_{3}}\right) n_{3}+K_{13}\left(y_{2}-y^{*}\right) \\
& W_{31}=\left(C^{16(1)} \frac{\partial \rho^{*}}{\partial x_{1}}+C^{66(1)} \frac{\partial \varrho^{*}}{\partial x_{2}}+C^{56(1)} \frac{\partial \rho^{*}}{\partial x_{3}}\right) n_{1}+\left(C^{12(1)} \frac{\partial \rho^{*}}{\partial x_{1}}+C^{26(1)} \frac{\partial \varrho^{*}}{\partial x_{2}}+C^{25(1)} \frac{\partial \rho^{*}}{\partial x_{3}}\right) n_{2}+\left(C^{14(1)} \frac{\partial \rho^{*}}{\partial x_{1}}+C^{46(1)} \frac{\partial \rho^{*}}{\partial x_{2}}+C^{45(1)} \frac{\partial \rho^{*}}{\partial x_{3}}\right) n_{3}+K_{21}\left(y^{*}-y_{1}\right) \\
& W_{32}=\left(C^{66(1)} \frac{\partial \rho^{*}}{\partial x_{1}}+C^{26(1)} \frac{\partial \varrho^{*}}{\partial x_{2}}+C^{46(1)} \frac{\partial \varrho^{*}}{\partial x_{3}}\right) n_{1}+\left(C^{26(1)} \frac{\partial \varrho^{*}}{\partial x_{1}}+C^{22(1)} \frac{\partial \varrho^{*}}{\partial x_{2}}+C^{24(1)} \frac{\partial \varrho^{*}}{\partial x_{3}}\right) n_{2}+\left(C^{46(1)} \frac{\partial \varrho^{*}}{\partial x_{1}}+C^{24(1)} \frac{\partial \rho^{*}}{\partial x_{2}}+C^{44(1)} \frac{\partial \rho^{*}}{\partial x_{3}}\right) n_{3}+K_{22}\left(y^{*}-y_{1}\right)
\end{aligned}
$$




$$
\begin{aligned}
& W_{33}=\left(C^{56(1)} \frac{\partial \rho^{*}}{\partial x_{1}}+C^{46(1)} \frac{\partial \varrho^{*}}{\partial x_{2}}+C^{36(1)} \frac{\partial \rho^{*}}{\partial x_{3}}\right) n_{1}+\left(C^{25(1)} \frac{\partial \varrho^{*}}{\partial x_{1}}+C^{24(1)} \frac{\partial \rho^{*}}{\partial x_{2}}+C^{23(1)} \frac{\partial \rho^{*}}{\partial x_{3}}\right) n_{2}+\left(C^{45(1)} \frac{\partial \rho^{*}}{\partial x_{1}}+C^{44(1)} \frac{\partial \varrho^{*}}{\partial x_{2}}+C^{34(1)} \frac{\partial \rho^{*}}{\partial x_{3}}\right) n_{3}+K_{23}\left(y^{*}-y_{1}\right) \\
& W_{34}=K_{21}\left(y_{2}-y^{*}\right) \quad W_{35}=K_{22}\left(y_{2}-y^{*}\right) \quad W_{36}=K_{23}\left(y_{2}-y^{*}\right) \\
& W_{41}=K_{21}\left(y^{*}-y_{1}\right) \quad W_{42}=K_{22}\left(y^{*}-y_{1}\right) \quad W_{43}=K_{23}\left(y^{*}-y_{1}\right) \\
& W_{44}=\left(C^{16(2)} \frac{\partial \rho^{*}}{\partial x_{1}}+C^{66(2)} \frac{\partial \rho^{*}}{\partial x_{2}}+C^{56(2)} \frac{\partial \rho^{*}}{\partial x_{3}}\right) n_{1}+\left(C^{12(2)} \frac{\partial \rho^{*}}{\partial x_{1}}+C^{26(2)} \frac{\partial \rho^{*}}{\partial x_{2}}+C^{25(2)} \frac{\partial \rho^{*}}{\partial x_{3}}\right) n_{2}+\left(C^{14(2)} \frac{\partial \rho^{*}}{\partial x_{1}}+C^{46(2)} \frac{\partial \rho^{*}}{\partial x_{2}}+C^{45(2)} \frac{\partial \rho^{*}}{\partial x_{3}}\right) n_{3}+K_{21}\left(y_{2}-y^{*}\right) \\
& W_{45}=\left(C^{66(2)} \frac{\partial \rho^{*}}{\partial x_{1}}+C^{26(2)} \frac{\partial \rho^{*}}{\partial x_{2}}+C^{46(2)} \frac{\partial \rho^{*}}{\partial x_{3}}\right) n_{1}+\left(C^{26(2)} \frac{\partial \rho^{*}}{\partial x_{1}}+C^{22(2)} \frac{\partial \rho^{*}}{\partial x_{2}}+C^{24(2)} \frac{\partial \rho^{*}}{\partial x_{3}}\right) n_{2}+\left(C^{46(2)} \frac{\partial \rho^{*}}{\partial x_{1}}+C^{24(2)} \frac{\partial \rho^{*}}{\partial x_{2}}+C^{44(2)} \frac{\partial \rho^{*}}{\partial x_{3}}\right) n_{3}+K_{22}\left(y_{2}-y^{*}\right) \\
& W_{46}=\left(C^{56(2)} \frac{\partial \rho^{*}}{\partial x_{1}}+C^{46(2)} \frac{\partial \rho^{*}}{\partial x_{2}}+C^{36(2)} \frac{\partial \rho^{*}}{\partial x_{3}}\right) n_{1}+\left(C^{25(2)} \frac{\partial \rho^{*}}{\partial x_{1}}+C^{24(2)} \frac{\partial \rho^{*}}{\partial x_{2}}+C^{23(2)} \frac{\partial \varrho^{*}}{\partial x_{3}}\right) n_{2}+\left(C^{45(2)} \frac{\partial \rho^{*}}{\partial x_{1}}+C^{44(2)} \frac{\partial \rho^{*}}{\partial x_{2}}+C^{34(2)} \frac{\partial \rho^{*}}{\partial x_{3}}\right) n_{3}+K_{23}\left(y_{2}-y^{*}\right) \\
& W_{51}=\left(C^{15(1)} \frac{\partial \rho^{*}}{\partial x_{1}}+C^{56(1)} \frac{\partial \rho^{*}}{\partial x_{2}}+C^{55(1)} \frac{\partial \rho^{*}}{\partial x_{3}}\right) n_{1}+\left(C^{14(1)} \frac{\partial \rho^{*}}{\partial x_{1}}+C^{46(1)} \frac{\partial \rho^{*}}{\partial x_{2}}+C^{45(1)} \frac{\partial \rho^{*}}{\partial x_{3}}\right) n_{2}+\left(C^{13(1)} \frac{\partial \rho^{*}}{\partial x_{1}}+C^{36(1)} \frac{\partial \rho^{*}}{\partial x_{2}}+C^{35(1)} \frac{\partial \rho^{*}}{\partial x_{3}}\right) n_{3}+K_{31}\left(y^{*}-y_{1}\right) \\
& W_{52}=\left(C^{56(1)} \frac{\partial \rho^{*}}{\partial x_{1}}+C^{25(1)} \frac{\partial \rho^{*}}{\partial x_{2}}+C^{45(1)} \frac{\partial \rho^{*}}{\partial x_{3}}\right) n_{1}+\left(C^{46(1)} \frac{\partial \rho^{*}}{\partial x_{1}}+C^{24(1)} \frac{\partial \rho^{*}}{\partial x_{2}}+C^{44(1)} \frac{\partial \rho^{*}}{\partial x_{3}}\right) n_{2}+\left(C^{36(1)} \frac{\partial \rho^{*}}{\partial x_{1}}+C^{23(1)} \frac{\partial \rho^{*}}{\partial x_{2}}+C^{34(1)} \frac{\partial \rho^{*}}{\partial x_{3}}\right) n_{3}+K_{32}\left(y^{*}-y_{1}\right) \\
& W_{53}=\left(C^{55(1)} \frac{\partial \rho^{*}}{\partial x_{1}}+C^{45(1)} \frac{\partial \varrho^{*}}{\partial x_{2}}+C^{35(1)} \frac{\partial \rho^{*}}{\partial x_{3}}\right) n_{1}+\left(C^{45(1)} \frac{\partial \rho^{*}}{\partial x_{1}}+C^{44(1)} \frac{\partial \varrho^{*}}{\partial x_{2}}+C^{34(1)} \frac{\partial \varrho^{*}}{\partial x_{3}}\right) n_{2}+\left(C^{35(1)} \frac{\partial \varrho^{*}}{\partial x_{1}}+C^{34(1)} \frac{\partial \rho^{*}}{\partial x_{2}}+C^{33(1)} \frac{\partial \rho^{*}}{\partial x_{3}}\right) n_{3}+K_{33}\left(y^{*}-y_{1}\right) \\
& W_{54}=K_{31}\left(y_{2}-y^{*}\right) \quad W_{55}=K_{32}\left(y_{2}-y^{*}\right) \quad W_{56}=K_{33}\left(y_{2}-y^{*}\right) \\
& W_{61}=K_{31}\left(y^{*}-y_{1}\right) \quad W_{62}=K_{32}\left(y^{*}-y_{1}\right) \quad W_{63}=K_{33}\left(y^{*}-y_{1}\right) \\
& W_{64}=\left(C^{15(2)} \frac{\partial \rho^{*}}{\partial x_{1}}+C^{56(2)} \frac{\partial \rho^{*}}{\partial x_{2}}+C^{55(2)} \frac{\partial \rho^{*}}{\partial x_{3}}\right) n_{1}+\left(C^{14(2)} \frac{\partial \rho^{*}}{\partial x_{1}}+C^{46(2)} \frac{\partial \rho^{*}}{\partial x_{2}}+C^{45(2)} \frac{\partial \rho^{*}}{\partial x_{3}}\right) n_{2}+\left(C^{13(2)} \frac{\partial \rho^{*}}{\partial x_{1}}+C^{36(2)} \frac{\partial \rho^{*}}{\partial x_{2}}+C^{35(2)} \frac{\partial \rho^{*}}{\partial x_{3}}\right) n_{3}+K_{31}\left(y_{2}-y^{*}\right) \\
& W_{65}=\left(C^{56(2)} \frac{\partial \rho^{*}}{\partial x_{1}}+C^{25(2)} \frac{\partial \rho^{*}}{\partial x_{2}}+C^{45(2)} \frac{\partial \rho^{*}}{\partial x_{3}}\right) n_{1}+\left(C^{46(2)} \frac{\partial \rho^{*}}{\partial x_{1}}+C^{24(2)} \frac{\partial \rho^{*}}{\partial x_{2}}+C^{44(2)} \frac{\partial \rho^{*}}{\partial x_{3}}\right) n_{2}+\left(C^{36(2)} \frac{\partial \rho^{*}}{\partial x_{1}}+C^{23(2)} \frac{\partial \rho^{*}}{\partial x_{2}}+C^{34(2)} \frac{\partial \rho^{*}}{\partial x_{3}}\right) n_{3}+K_{32}\left(y_{2}-y^{*}\right) \\
& W_{66}=\left(C^{55(2)} \frac{\partial \varrho^{*}}{\partial x_{1}}+C^{45(2)} \frac{\partial \varrho^{*}}{\partial x_{2}}+C^{35(2)} \frac{\partial \varrho^{*}}{\partial x_{3}}\right) n_{1}+\left(C^{45(2)} \frac{\partial \varrho^{*}}{\partial x_{1}}+C^{44(2)} \frac{\partial \varrho^{*}}{\partial x_{2}}+C^{34(2)} \frac{\partial \varrho^{*}}{\partial x_{3}}\right) n_{2}+\left(C^{35(2)} \frac{\partial \rho^{*}}{\partial x_{1}}+C^{34(2)} \frac{\partial \varrho^{*}}{\partial x_{2}}+C^{33(2)} \frac{\partial \varrho^{*}}{\partial x_{3}}\right) n_{3}+K_{33}\left(y_{2}-y^{*}\right) \\
& P_{1}^{a}=-C^{1 a(1)} n_{1}-C^{6 a(1)} n_{2}-C^{5 a(1)} n_{3} \\
& P_{2}^{a}=-C^{1 a(2)} n_{1}-C^{6 a(2)} n_{2}-C^{5 a(2)} n_{3} \\
& P_{3}^{a}=-C^{6 a(1)} n_{1}-C^{2 a(1)} n_{2}-C^{4 a(1)} n_{3} \\
& P_{4}^{a}=-C^{6 a(2)} n_{1}-C^{2 a(2)} n_{2}-C^{4 a(2)} n_{3} \\
& P_{5}^{a}=-C^{1 a(1)} n_{1}-C^{4 a(1)} n_{2}-C^{3 a(1)} n_{3} \\
& P_{6}^{a}=-C^{1 a(2)} n_{1}-C^{4 a(2)} n_{2}-C^{3 a(2)} n_{3}
\end{aligned}
$$

\section{References}

[1] Sreenivasa Prasath S, Arockiarajan A. Effect of interphase and thermal environment on the effective properties of Macro-Fiber Composites (MFC). Compos Part B 2015;75:327-35. https://doi.org/10.1016/j.compositesb.2015.02.006.

[2] Chatzigeorgiou G, Seidel GD, Lagoudas DC. Effective mechanical properties of aligned fuzzy fiber composites. Compos Part B 2012;43:2577-93.

[3] Sevostianov I. On the thermal expansion of composite materials and cross-property connection between thermal expansion and thermal conductivity. Mech Mater 2012;45:20-33. https://doi.org/10.1016/j.mechmat.2011.10.001.

[4] Srinivas S, Li JY. The effective magnetoelectric coefficients of polycrystalline multiferroic composites. Acta Mater 2005;53(15):4135-42. https://doi.org/10.1016/j. actamat.2005.05.014.

[5] Lim TK, Kim JH. Thermo-elastic effects on shear correction factors for functionally graded beam. Compos Part B 2017;123:262-70. https://doi.org/10.1016/j. compositesb.2017.05.031.

[6] Viet NV, Wang Q, Kuo WS. Effective Young's modulus of carbon nanotube/epoxy composites. Compos Part B 2016;94:160-6. https://doi.org/10.1016/j.compositesb. 2016.03.060.

[7] Amirpour M, Das R, Saavedra Flores EI. Analytical solutions for elastic deformation of functionally graded thick plates with in-plane stiffness variation using higher order shear deformation theory. Compos Part B 2016;94:109-21. https://doi.org/ 10.1016/j.compositesb.2016.03.040.

[8] Eigenstrain and Fourier series for evaluation of elastic local fields and effective properties of periodic composites, Compos Part B 81 (2015): 251-58. doi:10.1016/j. compositesb.2015.07.002.

[9] Tsalis D, Charalambakis N, Bonnay K, Chatzigeorgiou G. Effective properties of multiphase composites made of elastic materials with hierarchical structure. Math Mech Solids 2017;22(4):761-70. https://doi.org/10.1177/1081286515612142.

[10] Tsalis D, Bonnay K, Chatzigeorgiou G, Charalambakis N. Multiscale homogenization of multilayered structures. In: 8th GRACM International congress on computational mechanics (July).

[11] Pobedrya B. Mechanics of composite materials. Russia, Moscow: Moscow State University Press; 1984.

[12] Civalek Ö. An efficient method for free vibration analysis of rotating truncated conical shells. Int J Press Vessels Pip 2006;83(1):1-12. https://doi.org/10.1016/j. ijpvp.2005.10.005.

[13] Qian LF, Batra RC, Chen LM. Static and dynamic deformations of thick functionally graded elastic plates by using higher-order shear and normal deformable plate theory and meshless local Petrov-Galerkin method. Compos Part B 2004;35(6-8):685-97. https://doi.org/10.1016/j.compositesb.2004.02.004.

[14] Alibeigloo A. Effect of viscoelastic interface on three-dimensional static and vibration behavior of laminated composite plate. Compos Part B 2015;75:17-28. https://doi.org/10.1016/j.compositesb.2015.01.025.

[15] Tsalis D, Baxevanis T, Chatzigeorgiou G, Charalambakis N. Homogenization of 
elastoplastic composites with generalized periodicity in the microstructure. Int $\mathrm{J}$ Plast 2013;51:161-87.

[16] Tsalis D, Chatzigeorgiou G, Charalambakis N. Effective behavior of thermo-elastic tubes with wavy layers. Compos Part B 2016;99:173-87. https://doi.org/10.1016/j. compositesb.2016.05.043.

[17] Han J-H, Kardomateas GA, Simitses GJ. Elasticity, shell theory and finite element results for the buckling of long sandwich cylindrical shells under external pressure. Compos Part B 2004;35:591-8. https://doi.org/10.1016/j.compositesb.2003.07. 002.

[18] Tsukrov I, Drach B, Gross TS. Effective stiffness and thermal expansion coefficients of unidirectional composites with fibers surrounded by cylindrically orthotropic matrix layers. Int J Eng Sci 2012;58:129-43. https://doi.org/10.1016/j.ijengsci. 2012.03.032.

[19] Christensen RM. Effective properties for single size, rigid spherical inclusions in an elastic matrix. Compos Part B 2004;35(6-8):475-82. https://doi.org/10.1016/j compositesb.2004.04.008.

[20] Guinovart-Sanjuán D, Rizzoni R, Rodríguez-Ramos R, Guinovart-Díaz R, BravoCastillero J, Alfonso-Rodriguez R, et al. Assessment of models and methods for pressurized spherical composites. Math Mech Solids 2016;87:1-12. https://doi.org/ $10.1177 / 1081286516673233$.

[21] Sofiyev AH, Alizada AN, Akin Ö, Valiyev A, Avcar M, Adiguzel S. On the stability of FGM shells subjected to combined loads with different edge conditions and resting on elastic foundations. Acta Mech 2012;223(1):189-204. arXiv:84856063543, doi:10.1007/s00707-011-0548-1.

[22] Nazarenko L, Stolarski H. Energy-based definition of equivalent inhomogeneity for various interphase models and analysis of effective properties of particulate composites. Compos Part B 2016;94:82-94. https://doi.org/10.1016/j.compositesb. 2016.03.015.

[23] Vinh PC, Tung DX. Homogenization of rough two-dimensional interfaces separating two anisotropic solids. J Appl Mech 2011;78(4):041014. https://doi.org/10.1115/ 1.4003722.

[24] Vinh PC, Tung DX. Homogenization of very rough interfaces separating two piezoelectric solids. Acta Mech 2013;224(5):1077-88. https://doi.org/10.1007 s00707-012-0804-z.
[25] Kuo HY, Huang TY. Effective moduli of multiferroic fibrous composites with springtype imperfect interfaces under generalized plane strain with transverse electromagnetic fields. Int J Solids Struct 2016;80:456-64. https://doi.org/10.1016/j. ijsolstr.2015.10.007.

[26] Würkner M, Berger H, Gabbert U. Numerical study of effective elastic properties of fiber reinforced composites with rhombic cell arrangements and imperfect interface. Int J Eng Sci 2013;63:1-9. https://doi.org/10.1016/j.ijengsci.2012.10.002.

[27] Zhu QZ, Gu S, Yvonnet J, Shao J, He Q. Three-dimensional numerical modelling by XFEM of spring-layer imperfect curved interfaces with applications to linearly elastic composite materials. Proceedings of the 2011 American control conference. 88. 2011. p. 307-28.

[28] Medeiros RD, Moreno ME, Marques FD, Tita V. Effective properties evaluation for smart composite materials. J Brazilian Soc Mech Sci Eng 2012;34:362-70. https:// doi.org/10.1590/S1678-58782012000500004.

[29] Civalek Ö. Numerical analysis of free vibrations of laminated composite conical and cylindrical shells: discrete singular convolution (DSC) approach. J Comput Appl Math 2007;205(1):251-71. https://doi.org/10.1016/j.cam.2006.05.001.

[30] Sofiyev A, Aksogan O, Schnack E, Avcar M. The stability of a three-layered composite conical shell containing a FGM layer subjected to external pressure. Mech Adv Mater Struct 2008;15(6-7):461-6. https://doi.org/10.1080/ 15376490802138492.

[31] Sofiyev AH, Avcar M. The stability of cylindrical shells containing an FGM layer subjected to axial load on the pasternak foundation. Engineering 2010;02(04):228-36. https://doi.org/10.4236/eng.2010.24033.

[32] Guinovart-Sanjuán D, Rodríguez-Ramos R, Guinovart-Díaz R, Bravo-Castillero J, Sabina FJ, Merodio J, et al. Effective properties of regular elastic laminated shell composite. Compos Part B 2016;87:12-20. https://doi.org/10.1016/j.compositesb. 2015.09.051.

[33] Tsalis D, Chatzigeorgiou G, Charalambakis N. Homogenization of structures with generalized periodicity. Compos Part B 2012;43(2012):2495-512.

[34] Guinovart-Sanjuán D, Rizzoni R, Rodríguez-Ramos R, Guinovart-Díaz R, BravoCastillero J, Alfonso-Rodríguez R, Lebon F, et al. Behavior of laminated shell composite with imperfect contact between the layers. Compos Struct 2017;176. https://doi.org/10.1016/j.compstruct.2017.05.058. 